\title{
A Newly Synthesized Flavone from Luteolin Escapes from COMT-Catalyzed Methylation and Inhibits Lipopolysaccharide-Induced Inflammation in RAW264.7 Macrophages via JNK, p38 and NF-KB Signaling Pathways
}

\author{
Lin Ye ${ }^{1,2}$, Yang Xin ${ }^{3}$, Zhi-yuan Wu ${ }^{2}$, Hai-jian Sun ${ }^{2}$, De-jian Huang ${ }^{3,4}$, and Zhi-qin Sun ${ }^{5,1 *}$ \\ ${ }^{1}$ School of Pharmacy, Changzhou University, Changzhou 213164, P.R. China \\ ${ }^{2}$ Department of Pharmacology, Yong Loo Lin School of Medicine, National University of Singapore, Singapore \\ 117597, Singapore \\ ${ }^{3}$ Food Science and Technology Program, Department of Chemistry, Faculty of Science, National University of \\ Singapore, Singapore 117597, Singapore \\ ${ }^{4}$ National University of Singapore (Suzhou) Research Institute, Suzhou, Jiangsu 215123, P.R. China \\ ${ }^{5}$ Changzhou Second People's Hospital, Changzhou 213000, P.R. China
}

Luteolin is a common dietary flavone possessing potent anti-inflammatory activities. However, when administrated in vivo, luteolin becomes methylated by catechol-0-methyltransferases (COMT) owing to the catechol ring in the chemical structure, which largely diminishes its anti-inflammatory effect. In this study, we made a modification on luteolin, named LUA, which was generated by the chemical reaction between luteolin and 2,2'-azobis(2-amidinopropane) dihydrochloride (AAPH). Without a catechol ring in the chemical structure, this new flavone could escape from the COMTcatalyzed methylation, thus affording the potential to exert its functions in the original form when administrated in the organism. Moreover, an LPS-stimulated RAW cell model was applied to detect the anti-inflammatory properties. LUA showed much more superior inhibitory effect on LPS-induced production of NO than diosmetin (a major methylated form of luteolin) and significantly suppressed upregulation of iNOS and COX-2 in macrophages. LUA treatment dramatically reduced LPSstimulated reactive oxygen species (ROS) and mRNA levels of pro-inflammatory mediators such as IL-1 $\beta$, IL-6, IL-8 and IFN- $\beta$. Furthermore, LUA significantly reduced the phosphorylation of JNK and p38 without affecting that of ERK. LUA also inhibited the activation of NF-KB through suppression of p65 phosphorylation and nuclear translocation.

Keywords: Luteolin, 2,2'-azobis(2-amidinopropane) dihydrochloride (AAPH), catechol-O-methyltransferases (COMT), inflammation

Received: April 20, 2021 Accepted: May 25, 2021

First published online: May 27,202

* Corresponding author Phone: +13861285688 E-mail:sun_zhiqin@163.com

pISSN 1017-7825 eISSN 1738-8872

Copyright $@ 2022$ by the authors. Licensee KMB. This article is an open access article distributed under the terms and conditions of the Creative Commons Attribution (CC BY) license

\section{Introduction}

Inflammatory response, as a common host defense reaction against pathogen invasion or other injuries, plays a crucial role in the protection of vascularized living organisms. Normally, inflammation is accompanied by various kinds of processes, among which the innate immune system is initially activated. Macrophages are the primary innate immune cells substantially responsible for phagocytosis and antigen presentation $[1,2]$. When the macrophages are stimulated by harmful factors, they can produce and release many inflammation mediators and cytokines like nitric oxide (NO), prostaglandins (PGs), tumor necrosis factor- $\alpha$ (TNF- $\alpha$ ), interleukin- $1 \beta$ (IL- $1 \beta$ ), and interleukin-6 (IL-6). Although moderate inflammation contributes to the elimination of microbes from tissues [3], excessive activation of inflammation has been identified to induce cell damages and even some chronic diseases based on inflammation including atherosclerosis, diabetes, rheumatoid arthritis, and cancer [4-7]. Therefore, therapeutic drugs with anti-inflammatory properties are required to alleviate damage induced by excessive inflammatory response.

Flavones are a class of flavonoids typically found in a variety of fruits and vegetables [8] and have been employed as anti-oxidative, anti-inflammatory, anti-microbial, anti-diabetic and immunoregulatory remedies for centuries [9-12]. Luteolin (3',4',5,7-hydroxyl-flavone, Fig. 1), a natural flavone abundant in vegetables such as cabbages, celery, carrots, broccoli, peppers, onion leaves, apple skins, and chrysanthemum flowers [13], has received 
<smiles>O=c1cc(-c2ccc(O)c(O)c2)oc2cc(O)cc(O)c12</smiles>

LUT

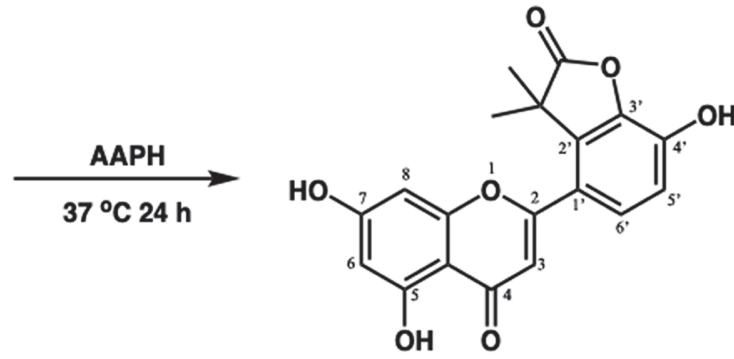

LUA

Fig. 1. Synthetic pathway and chemical structure of LUA. LUA: LUAAPH-1.

extensive attention not only because of its versatile health benefits [14-17], but also for the fact that it exerts pharmacological activity at micromolar concentrations [18]. Increasing evidence has demonstrated that luteolin exhibits one of the strongest anti-inflammatory properties among flavonoids via different mechanisms both in vivo and in vitro [14, 19-21].

However, despite the intense anti-inflammation activity, the bioactivity of luteolin will decrease when administrated in vivo [22]. This may be explained partly because of the wide methylation of luteolin in the body, which is mediated by catechol-O-methyltransferases (COMT). In mammals, COMT is an enzyme ubiquitously distributed throughout the organs of the body. The highest concentration of COMT protein and activity levels is observed in the liver, followed by the kidneys and gastrointestinal tract [23-26]. COMT can catalyze the Omethylation of any substrate containing the structure of catechol, such as epinephrine, norepinephrine, and dopamine [27]. Luteolin, with a catechol ring structure, has also been identified as a substrate of COMT [28, 29]. Numerous studies have detected the methylated metabolites of luteolin, which are composed of diosmetin, chrysoeriol and their respective glucuronides conjugates [30,31]. The methylated metabolites not only lead to the faster elimination of luteolin [22], but also have much weaker anti-inflammatory effects than luteolin [31]. However, in the presence of COMT inhibitor, methylated derivatives of luteolin are remarkably reduced, leaving more luteolin conjugates metabolites exhibiting superior anti-inflammatory activities [31]. These results demonstrate that the methylation by COMT plays a critical role in inhibiting the anti-inflammatory effects of luteolin in the body. Therefore, it is necessary to prevent luteolin from being methylated by COMT to preserve its anti-inflammatory activities. Although COMT inhibitors have been discovered, some of them are associated with lots of problems, such as cytotoxicity, low bioavailability, short-acting inhibitory profile and intestinal side effects [32-34]. For this reason, there is a requirement for safer and more efficacious approaches to improve the methylated disposition of luteolin.

To achieve this objective, we sought to optimize the structure of luteolin by chemical methods, generating a new flavone compound named LUAAPH-1(LUA), which would escape methylation by COMT and exert strong antiinflammation effects. In vitro studies were then performed on RAW264.7 cell lines to detect the antiinflammatory activity of the new compound.

\section{Materials and Methods}

\section{Reagents and Antibodies}

Lipopolysaccharide (LPS, Escherichia coli 0111:B4) and 2,7-dichlorofluorescein diacetate (DCFH-DA) were obtained from Sigma-Aldrich (USA). Water-soluble tetrazolium-1(WST-1) for cell proliferation analysis was from BioVision (USA). Griess reagent was supplied by Promega (USA). The antibody for COX-2, $t$-P65, $\beta$-actin, and GAPDH was from Santa Cruz Biotechnology (USA). The IL-1 $\beta$ antibody was from Abcam (USA). All the other antibodies were obtained from Cell Signaling Technology (USA). Any other reagent was obtained from Sigma-Aldrich unless specifically stated. Luteolin was supplied by Nanjing Plant Origin Biological Technology Co., Ltd. Lastly, 2,2'-azobis(2-amidinopropane) dihydrochloride (AAPH) was obtained from Merck \& Co., Inc. (USA).

\section{The Synthesis Procedure of the New Flavone}

LUA was synthesized and identified in our laboratory previously. Briefly, 2,2'-azobis(2-amidinopropane) dihydrochloride (AAPH, $244 \mathrm{mg}, 0.9 \mathrm{mmol}$ ) and luteolin $(42.9 \mathrm{mg}, 0.15 \mathrm{mmol}$ ) were dissolved in $75 \mathrm{mM}$ phosphate buffer ( $\mathrm{pH} 7.4$ ) in a $50 \mathrm{ml}$ round-bottom flask. The mixture was stirred at $37^{\circ} \mathrm{C}$ under $\mathrm{O}_{2}$ for 24 h before the volatiles were evaporated to dryness on a rotary evaporator. The solid was purified on silica gel chromatography eluted with EtOAc/methanol $(10: 1 \mathrm{v} / \mathrm{v}, \mathrm{Rf}=0.35$ by TLC $)$. After workup, the desired product was obtained with yield of $20.2 \mathrm{mg}$ (47.1\%). ESI-MS: 353.07 (M-) (Fig. 1).

\section{Cell Culture}

Murine RAW264.7 macrophages obtained from the American Type Culture Collection (ATCC) were cultured in a-MEM containing 10\% FBS (heat-inactivated) supplemented with $1 \%$ penicillin/streptomycin (Hyclone Laboratories, USA). Cells were seeded to culture plates at a density of $10^{6}$ cells $/ \mathrm{ml}$ and grown in a humidified chamber with $95 \%$ air and $5 \% \mathrm{CO}_{2}$ at $37^{\circ} \mathrm{C}$ for at least $12 \mathrm{~h}$ to make them attach to the dishes. After that, the cells 
Table 1. Primers for real-time RT-PCR [78].

\begin{tabular}{|c|c|}
\hline Name & Sequences $\left(5^{\prime} \rightarrow 3^{\prime}\right)$ \\
\hline GAPDH & $\begin{array}{l}\text { Forward: ACCCCAGCAAGGACACTGAGCAAG } \\
\text { Reverse: GGCCCCTCCTGTTATTATGGGGGT }\end{array}$ \\
\hline iNOS & $\begin{array}{l}\text { Forward: GCTCGCTTTGCCACGGACGA } \\
\text { Reverse: AAGGCAGCGGGCACATGCAA }\end{array}$ \\
\hline COX-2 & $\begin{array}{l}\text { Forward: GGGCTCAGCCAGGCAGCAAAT } \\
\text { Reverse: GCACTGTGTTTGGGGTGGGCT }\end{array}$ \\
\hline IL-1 $\beta$ & $\begin{array}{l}\text { Forward: GCCTCGTGCTGTCGGACCCATAT } \\
\text { Reverse: TCCTTTGAGGCCCAAGGCCACA }\end{array}$ \\
\hline IL-6 & $\begin{array}{l}\text { Forward: AGACAAAGCCAGAGTCCTTCAGAGA } \\
\text { Reverse: GCCACTCCTTCTGTGACTCCAGC }\end{array}$ \\
\hline IL-8 & $\begin{array}{l}\text { Forward: TTGCCTTGACCCTGAAGCCCCC } \\
\text { Reverse: GGCACATCAGGTACGATCCAGGC }\end{array}$ \\
\hline IFN- $\beta$ & $\begin{array}{l}\text { Forward: GGATCCTCCACGCTGCGTTCC } \\
\text { Reverse: CCGCCCTGTAGGTGAGGTTGA }\end{array}$ \\
\hline
\end{tabular}

were stimulated with LPS $(200 \mathrm{ng} / \mathrm{ml})$ in the absence or presence of various compounds (dissolved in $0.2 \%$ DMSO).

\section{Cell Viability Assay}

Reagent WST-1 was applied to test cell proliferation in accordance with the manufacturer's instructions. Briefly, the cells grown in 96-well plates were exposed to various concentrations of indicated drugs for $24 \mathrm{~h}$. Afterward, the supernatant was replaced by fresh culture medium containing 10\% WST-1. After incubating for an additional $1 \mathrm{~h}$, the optical density was determined using a Varioskan Flash microplate reader (USA) at $440 \mathrm{~nm}$.

\section{NO Detection}

Cells grown in triplicate in 96-well plates were stimulated with or without LPS in the absence or presence of related compounds for $24 \mathrm{~h}$, after which the production of $\mathrm{NO}$ was measured using the Griess reagent [sulfanilamide solution, N-1-napthylethylenediamine dihydrochloride (NED) solution, standard nitrite sample]. In brief, the cell culture supernatant $(50 \mu \mathrm{l})$ was transferred to another $96-$ well plate, followed by sequential allocation of $50 \mu \mathrm{l}$ sulfanilamide solution and equal volumes of NED solution. The combination was left at room temperature for 5-10 min and then the optical density was read at $540 \mathrm{~nm}$ with a Varioskan Flash microplate reader. The concentrations of nitrite were identified from a standard reference curve generated with serial dilutions of standard nitrite sample in culture medium.

\section{Real-Time RT-PCR Analysis}

The mRNA levels of inducible nitric oxide synthase (iNOS), cyclooxygenase-2 (COX-2), IL-1 $\beta$, IL-6, IL-8, and interferon- $\beta$ (IFN- $\beta$ ) were detected by real-time reverse transcriptase polymerase chain reaction (RT-PCR). After stimulating RAW cells in the 12-well tissue culture plates for $6 \mathrm{~h}$, TRIzol reagent (Invitrogen, USA) was used to isolate total RNA from cells and $1 \mu \mathrm{g}$ RNA was converted to cDNA using an iScriptTM cDNA Synthesis Kit (BioRad, USA) according to the protocols provided by the manufacturer. The cDNA was amplified in triplicate using the GoTaq qPCR Master Mix (Promega) on the ViiA 7 real-time PCR system (Life Technologies Corporation, USA). All the primers were obtained from Integrated DNA Technologies (USA) and the sequences were shown in Table 1 . The thermal and timing conditions were $95^{\circ} \mathrm{C}(5 \mathrm{~min})$, and 40 cycles of $95^{\circ} \mathrm{C}(15 \mathrm{~s})$ and $60^{\circ} \mathrm{C}(1 \mathrm{~min})$. The $\mathrm{Ct}$ values achieved by RT-PCR were normalized according to the level of GAPDH (internal control) and 2- $\Delta \Delta \mathrm{Ct}$ formula was used to determine their relative quantities.

\section{Western Blot Analysis}

Cells cultured in 6-well tissue culture plates were stimulated for $30 \mathrm{~min}$ to detect the protein expression level of mitogen-activated protein kinases (MAPKs) and NF- $\kappa$ B P65, while the iNOS, COX-2, and IL- $1 \beta$ protein levels were analyzed after $24 \mathrm{~h}$ treatment. Cells were then washed 3 times with chilled PBS after treatment and lysed with RIPA buffer supplemented with phosphatase and protease inhibitors (Thermo Fisher Scientific). The protein concentration was measured with the BCA Colorimetric Protein Assay Kit (USA). Equal concentrations of cellular protein were separated by 10 or $12 \%$ SDS-PAGE and then electro-blotted onto a PVDF membrane. After being blocked with $5 \%$ skim milk at room temperature for $1 \mathrm{~h}$, the membranes were incubated with primary antibody overnight at $4^{\circ} \mathrm{C}$. Then membranes were washed for three times with TBST (Tris-buffered saline, $0.1 \%$ Tween 20) buffer, followed by incubation with appropriate horseradish peroxidase-conjugated secondary antibody for $1 \mathrm{~h}$ at room temperature, and washed for 3 times in TBST again. The immunoblots were visualized with the WesternBright ECL Detector (Advansta, USA). The density of the protein bands was quantified by Image-Pro Plus and normalized with GAPDH, $\beta$-actin or non-phosphorylated form of protein levels.

\section{Reactive Oxygen Species (ROS) Assay}

The DCFH-DA method was used to measure intracellular ROS generation. After treatment with LPS for $24 \mathrm{~h}$, cells in the 96 -well plate were stained with $10 \mu \mathrm{M} \mathrm{DCFH}-\mathrm{DA}$ for $30 \mathrm{~min}$ at $37^{\circ} \mathrm{C}$. Dye fluorescence intensity was 
monitored at excitation $(485 \mathrm{~nm})$ and emission $(535 \mathrm{~nm})$. Fluorescent images were photographed using a Leica fluorescence microscope (Leica Microsystems, Germany) and fluorescence intensity was quantified by Image-Pro Plus.

Fluorescence Microscopy for p65 Nuclear Translocation

Cells were seeded on glass coverslips in a 24-well culture plate and stimulated for $24 \mathrm{~h}$. p 65 nuclear translocation was immunofluorescence-labeled using a cellular nuclear factor kappa B (NF- $\mathrm{kB}$ ) p65 translocation kit (Beyotime Biotech, China) in accordance with the manufacturer's instructions. Briefly, after fixing with stationary liquid for $10 \mathrm{~min}$, the cells were blocked for $1 \mathrm{~h}$ at room temperature and then incubated with the antibody against p65 overnight at $4^{\circ} \mathrm{C}$. The next day, the cells were washed with PBS and incubated with the rabbit IgG antibody conjugated with $\mathrm{Cy} 3$ for $1 \mathrm{~h}$ and stained with 2-(4-amidinophenyl)-6-indolecarbamidine dihydrochloride (DAPI) solution for $5 \mathrm{~min}$. Eventually, images were visualized via a laser confocal scanning microscope (Leica Microsystems) in the dark room at different excitation wavelengths ( $350 \mathrm{~nm}$ for DAPI and $540 \mathrm{~nm}$ for Cy3). The two images were combined together to confirm the areas of colocalization.

\section{Statistical Analysis}

The data were presented as mean \pm SD of at least three independent experiments and were analyzed via one-way analysis of variance (ANOVA) using Graphpad Prism 5 software. Differences were defined as statistically significant only for $p<0.05$.

\section{Results}

\section{Effect of LUA on Cell Viability in RAW Cells}

To observe the cytotoxic effect of LUA, RAW264.7 cells were exposed to different concentrations of LUA for 24 h. As shown in Fig. 2, LUA alone at 1-20 $\mu$ M showed no obvious changes in the cell viability assessed with the WST-1 assay.

\section{LUA Reduces LPS-Stimulated NO Production and iNOS Expression}

To test the effects of LUA on the LPS-induced inflammation, we first determined the concentration-dependent effect of LUA on NO levels in RAW264.7 cells in the presence or absence of LPS for $24 \mathrm{~h}$. As shown in Fig. 3A, LPS stimulation led to marked increases of NO levels, which was effectively suppressed by LUA $(0,1,5,10,15,20 \mu \mathrm{M})$ in a dose-dependent manner. LUA at $15 \mu \mathrm{M}$ significantly decreased NO by $50 \%$. The concentration of LUA at $15 \mu \mathrm{M}$ was therefore chosen in the subsequent experiments. We also compared the effects of luteolin, diosmetin and LUA on the production of NO. As shown in Fig. 3B, diosmetin had much weaker effect on suppression of NO production than luteolin and LUA. In accordance with NO production, treatment with LUA $(15 \mu \mathrm{M})$ also downregulated the LPS-induced upregulation of both mRNA (Fig. 3C) and protein (Fig. 3D) expression of iNOS.

\section{LUA Inhibits LPS-Induced COX-2 mRNA and Protein Expression}

To further confirm the anti-inflammatory effect of LUA, we determined the effect of LUA on the mRNA and protein expression of COX-2 in RAW264.7 cells stimulated with LPS for $6 \mathrm{~h}$ or $24 \mathrm{~h}$. As shown in Figs. 4A and 4B, stimulation with LPS remarkably increased COX-2 protein expression in RAW cells. However, co-treatment with LUA significantly attenuated LPS-induced COX-2 mRNA and protein expressions in the cells.

\section{LUA Regulates LPS-Induced Expression of Pro-Inflammatory Cytokines}

To evaluate the effect of LUA on pro-inflammatory cytokines, the mRNA levels of IL- $1 \beta$, IL-6, IL- 8 , and IFN- $\beta$ were examined by RT-PCR after treatment with LPS and LUA. As shown in Figs. 5A-5D, the mRNA expression of IL-1 $\beta$, IL-6, IL-8 and IFN- $\beta$ were significantly elevated at $6 \mathrm{~h}$ in response to LPS treatment. As expected, LUA

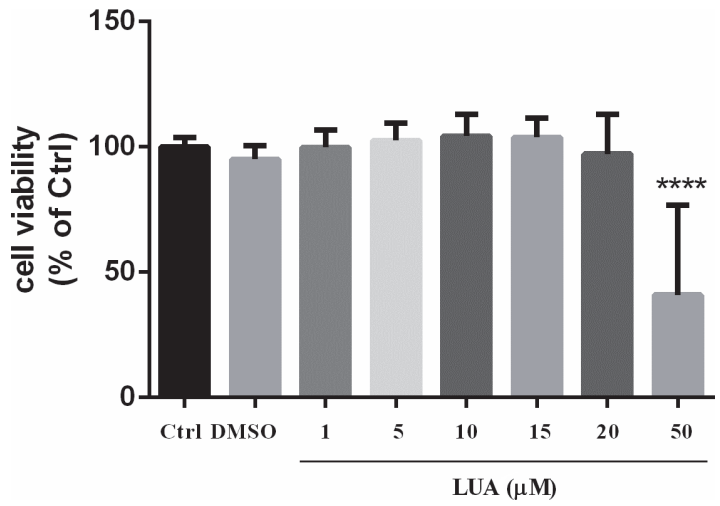

Fig. 2. Effect of LUA on cell viability in RAW267.4 cells. The cells were treated with $0.2 \%$ DMSO or various concentrations $(1-50 \mu \mathrm{M})$ of LUA for $24 \mathrm{~h}$. Cell viability was assessed using the WST-1 assay. Each value was presented as mean $\pm \mathrm{SD}(n=10) .{ }^{* * *} p<0.0001$ vs. $0.2 \%$ DMSO-treated cells. 

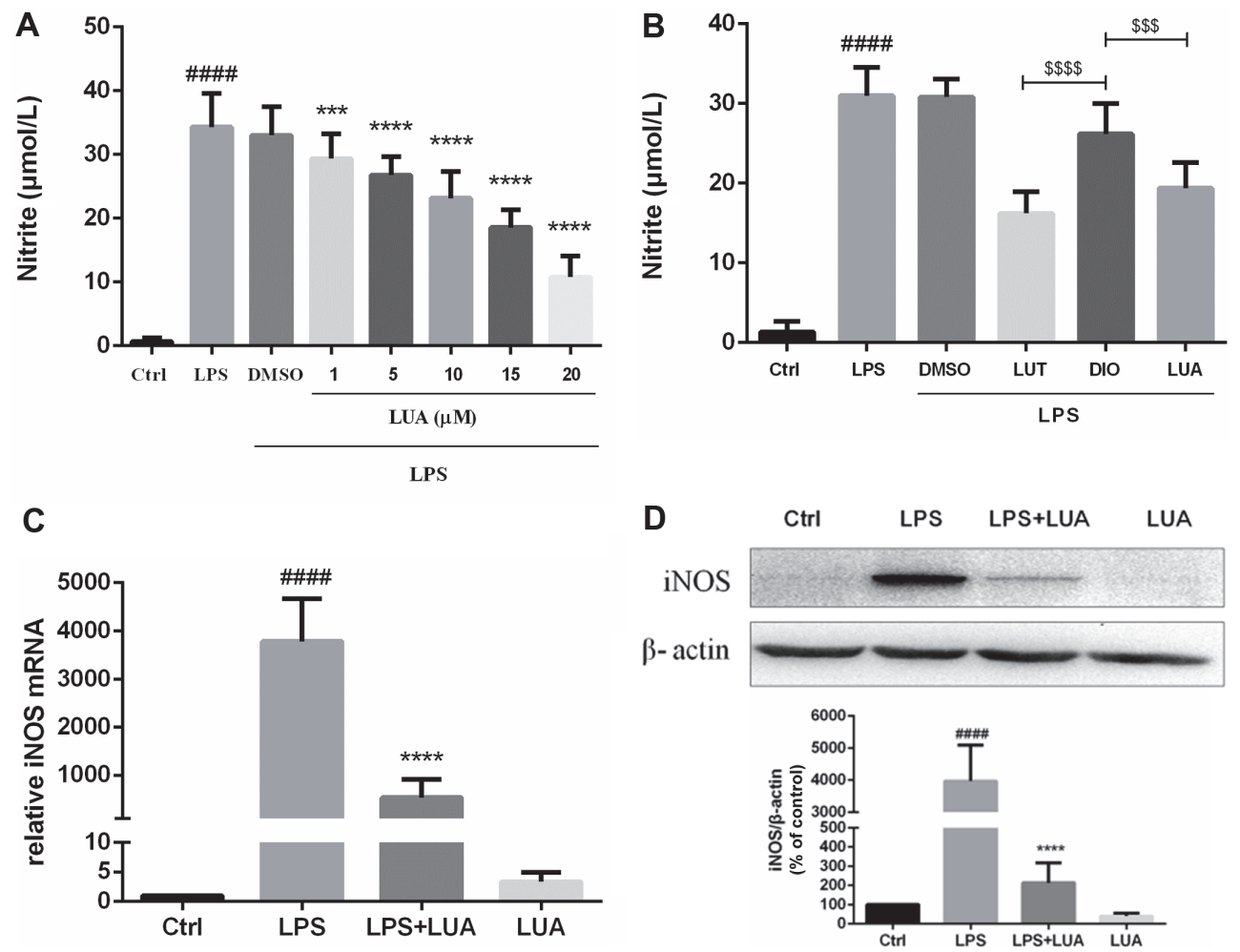

Fig. 3. LUA inhibited LPS-induced production of NO in Raw264.7 cells via the downregulation of iNOS expression. (A) Cells were incubated with various doses (1-20 $\mu \mathrm{mol} / \mathrm{l})$ of LUA in the absence or presence of LPS. The media were collected after $24 \mathrm{~h}$ and assayed for NO production $(n=13)$. (B) Cells were incubated with luteolin, diosmetin and LUA at the concentration of $15 \mathrm{uM}$ in the absence or presence of LPS. The media were collected after $24 \mathrm{~h}$ and assayed for NO $(n=4)$. (C and D) Cells were treated with LUA at the concentration of $15 \mathrm{uM}$ in the absence or presence of LPS. (C) After incubation for 6h, total RNA was isolated and RT-PCR was conducted for iNOS mRNA level $(n=3)$. (D) After incubation for $24 \mathrm{~h}$, cell lysates were subjected to Western blot analysis with an iNOS or $\beta$-actin-specific antibody $(n=5)$. The results were displayed as means \pm SD. Data were presented as percentage of control group. \#\#\#\#p<0.0001 vs. Ctrl group; ${ }^{* *} p<0.001,{ }^{* * *} p<0.0001$ vs. LPS group. Ctrl: control; LUT: luteolin; DIO: diosmetin; LUA: LUAAPH-1.

A

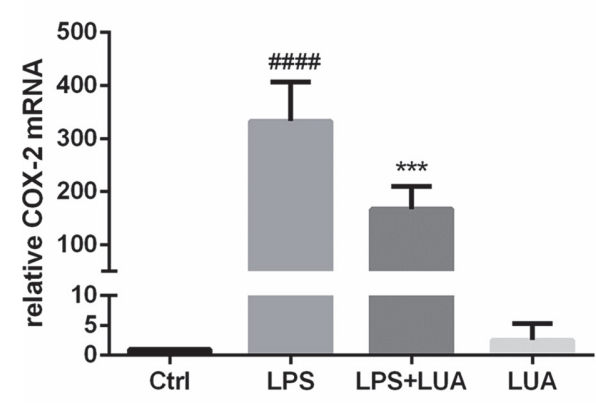

B

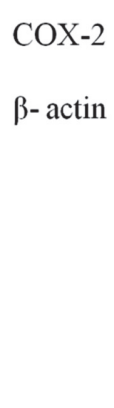

Ctrl LPS LPS+LUA LUA
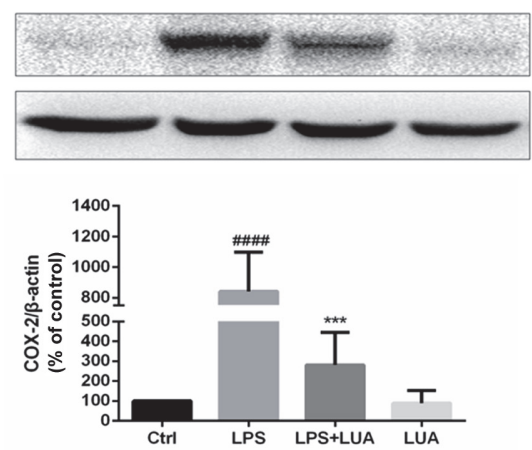

Fig. 4. Effects of LUA on LPS-induced COX-2 mRNA (A) and protein expression (B) in RAW264.7 cells. Cells were stimulated with or without LPS in the presence or absence of LUA $(15 \mu \mathrm{M})$. (A) The COX-2 mRNA expression level was detected by RT-PCR after treatment for $6 \mathrm{~h}(n=4)$. (B) The COX-2 protein expression level was measured by Western blot after stimulation for $24 \mathrm{~h}(n=4)$. Data were presented as percentage of control group. The results were displayed as means \pm SD. $\# \# \# p<0.0001$ vs. Ctrl group; ${ }^{* * *} p<0.001$ vs. LPS group. Ctrl: control; LUA: LUAAPH-1.

$(15 \mu \mathrm{M})$ attenuated LPS-induced upregulation of indicated mRNA levels. Moreover, the protein expression of IL$1 \beta$ was also analyzed at $24 \mathrm{~h}$ after LPS and LUA co-incubation. Consistent with RT-PCR results, treatment with LUA $(15 \mu \mathrm{M})$ suppressed LPS-induced increase in IL-1 $\beta$ protein expression, revealing that the reduction in IL- $1 \beta$ protein expression was associated with a decrease in the corresponding mRNA level (Fig. 5E). 
A

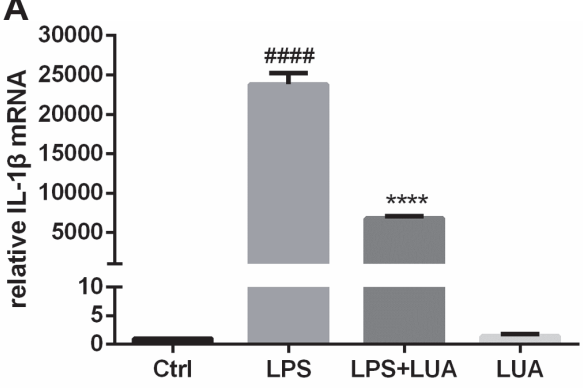

C

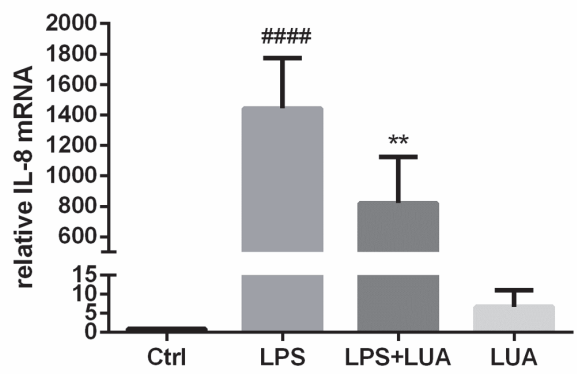

B

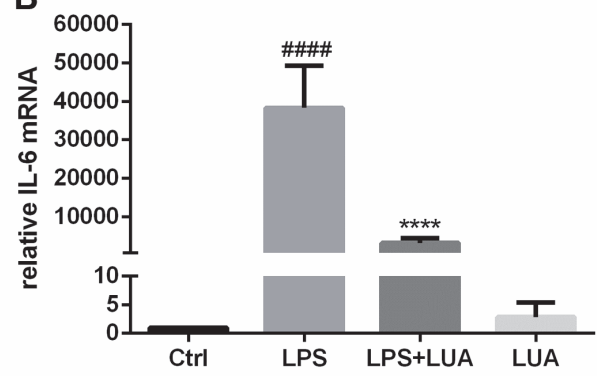

D

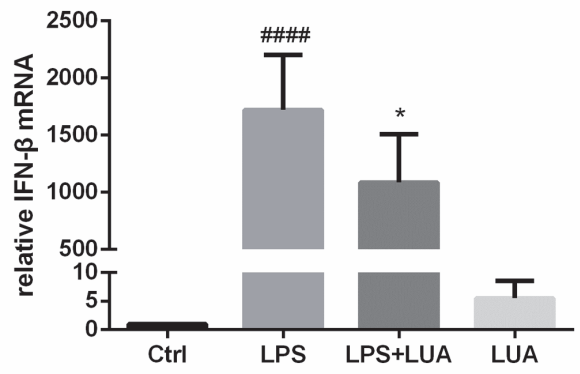

E

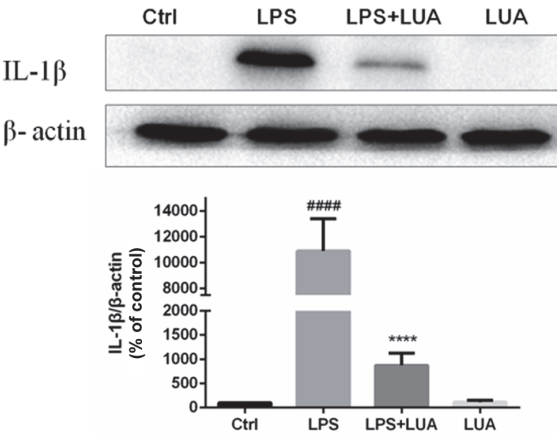

Fig. 5. Effects of LUA on LPS-induced pro-inflammatory cytokines in RAW264.7 cells. Cells were treated with LPS in the absence or presence of LUA (15 $\mu$ M). (A-D) Following stimulation with LPS for $6 \mathrm{~h}$, the IL-1 $\beta(\mathbf{A})(n=3)$, IL-6 (B) $(n=6)$, IL-8 (C) $(n=5)$, IFN- $\beta$ (D) $(n=4)$ mRNA expression levels were determined by RT-PCR. (E) Following incubation for $24 \mathrm{~h}$, the protein quantity of IL-1 $\beta$ was measured by Western blot $(n=4)$. Data were presented as percentage of control group. The results were displayed as means $\pm S D$. \#\#\#\# $p<0.0001$ vs. Ctrl group; ${ }^{*} p<0.05,{ }^{* *} p<0.01,{ }^{* * *} p<0.0001$ vs. LPS group. Ctrl: control; LUA: LUAAPH-1.

\section{LUA Suppresses LPS-Evoked Cellular ROS Generation}

ROS production is considered to be involved in the inflammation responses triggered by LPS in macrophages $[35,36]$. Therefore, the effect of LUA on intracellular ROS formation was monitored. As shown in Fig. 6, LPS treatment for $24 \mathrm{~h}$ induced massive production of intracellular ROS, which was dramatically reduced by the cotreatment with LUA $(15 \mu \mathrm{M})$.

\section{LUA Abolishes LPS-Triggered Phosphorylation of MAPKs}

MAPK signaling pathways play significant roles in the regulation of inflammatory responses [37-41]. Therefore, to investigate the effects of LUA on LPS-induced MAPK signaling, the levels of p-JNK, p-ERK and pp38 were measured in LPS-stimulated RAW264.7 cells. As shown in Figs. 7A and 7C, without altering total expression levels of MAPKs, treatment with LPS for 30 min resulted in the phosphorylation of JNK and p38, which were abrogated by LUA $(15 \mu \mathrm{M})$. However, the phosphorylation of ERK was not affected by LUA (Fig. 7B), indicating that LUA strongly blocked the JNK and p38 pathway but not ERK pathway in LPS-stimulated RAW cells.

\section{LUA Ameliorates LPS-Triggered p65 Phosphorylation and Nuclear Translocation}

NF- $\kappa \mathrm{B}$ is also critically involved in inflammatory processes. The effects of LUA on LPS-stimulated NF- $\kappa \mathrm{B}$ activation were shown in Fig. 8. As expected, the phosphorylated NF- $\mathrm{kB}$ p65 in RAW cells was markedly upregulated by LPS stimulation for $30 \mathrm{~min}$. In contrast, LUA $(15 \mu \mathrm{M})$ inhibited the expression of phosphorylated 


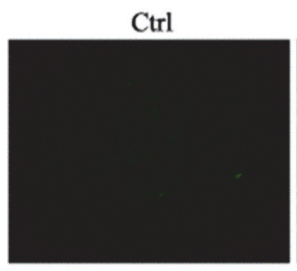

LPS+LUA

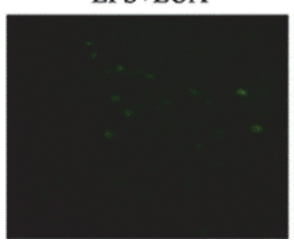

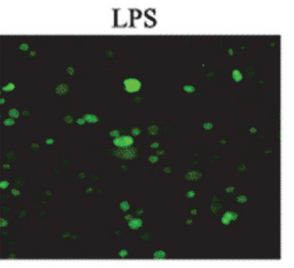

LUA

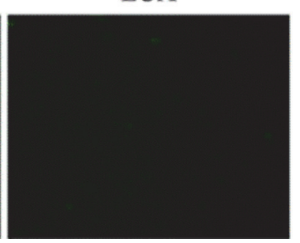

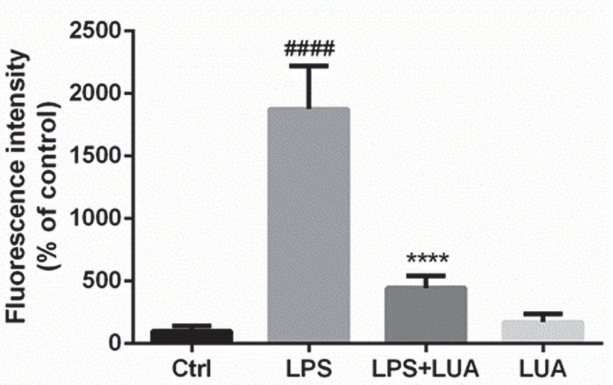

Fig. 6. Intracellular ROS levels in RAW264.7 cells. After incubating with LPS in the presence or absence of LUA $(15 \mu \mathrm{M})$ for $24 \mathrm{~h}$, the cells were exposed to DCFH-DA for $30 \mathrm{~min}$ at $37^{\circ} \mathrm{C}$. The fluorescence was measured at $485 \mathrm{~nm}$ (excitation) and $535 \mathrm{~nm}$ (emission). Data were presented as percentage of control group $(n=6)$. The results were displayed as means \pm SD. \#\#\#\# $<0.0001$ vs. Ctrl group; ${ }^{* * * *} p<0.0001$ vs. LPS group. Ctrl: control; LUA: LUAAPH-1.

A
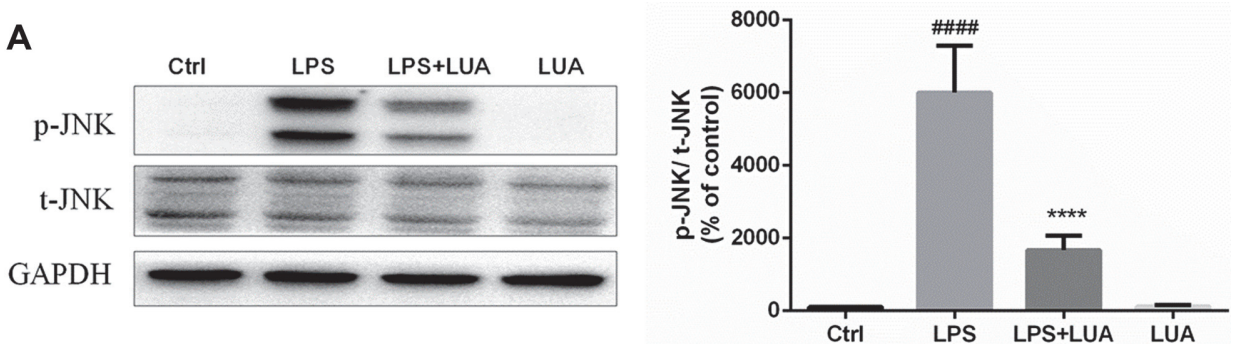

B
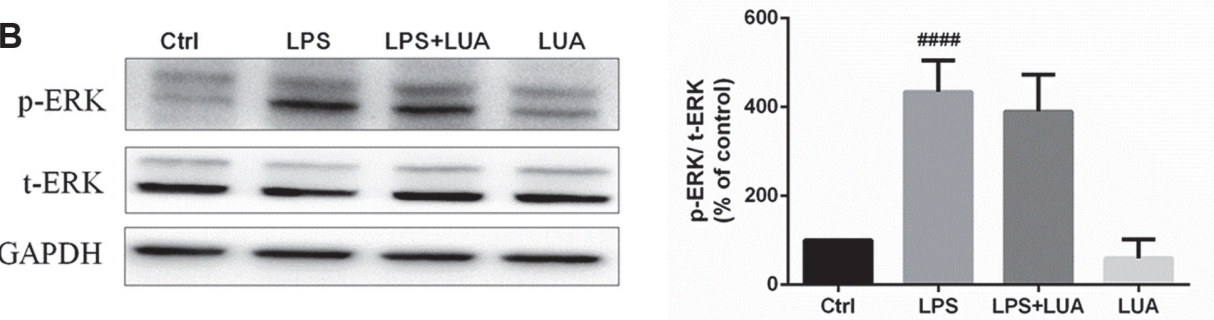

C
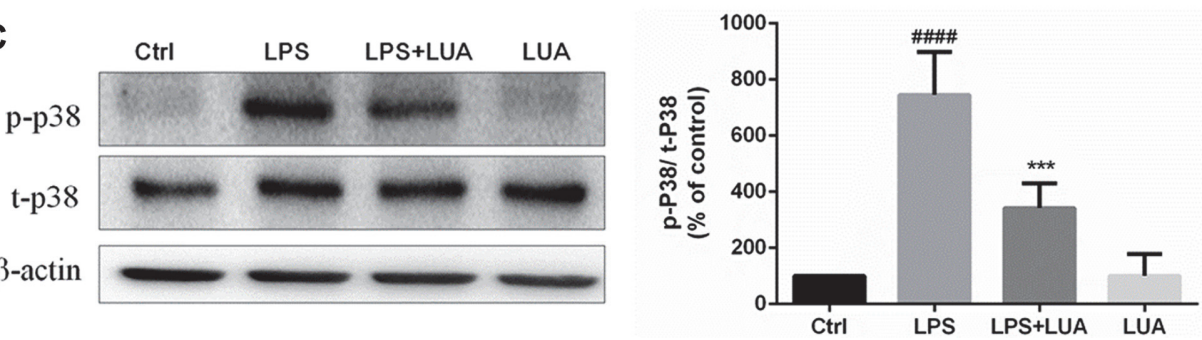

Fig. 7. Effects of LUA on LPS-induced MAPK signaling pathways in RAW264.7 cells. Cells were incubated with LPS for $30 \mathrm{~min}$ in the absence or presence of LUA $(15 \mu \mathrm{M})$. Cells lysates were subjected to Western blot for analysis of p-JNK (A) $(n=4)$, p-ERK (B) $(n=5)$ and p-P38 (C) $(n=4)$. The relative abundance of the phosphorylated form to its total protein was quantified. Data were presented as percentage of control group. The results were displayed as means \pm SD. \#\#\#\#p<0.0001 vs. Ctrl group; ${ }^{* * *} p<0.001,{ }^{* * * *} p<0.0001$ vs. LPS group. Ctrl: control; LUA: LUAAPH-1.

p65 challenged by LPS, with no significant difference of total p65 expression (Fig. 8A). In parallel, p65 nuclear translocation was measured by immunofluorescence using a confocal laser microscope. In line with previous results, the level of nuclear p65 was dramatically increased after LPS stimulation for $1 \mathrm{~h}$, which in turn was markedly suppressed by LUA $(15 \mu \mathrm{M})$ co-treatment (Fig. 8B). 
A

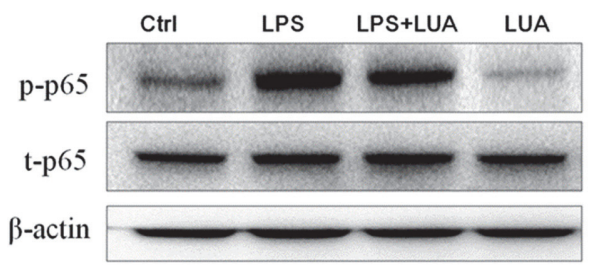

B
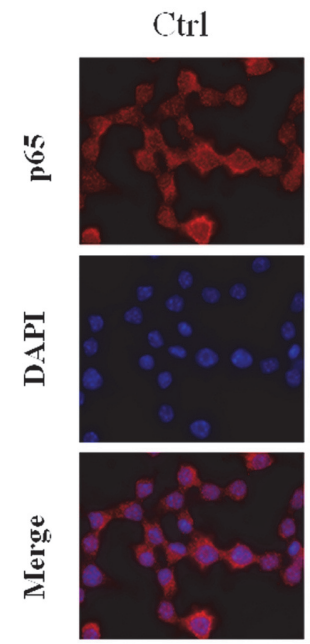

LPS
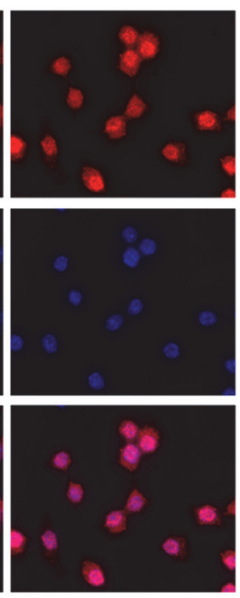

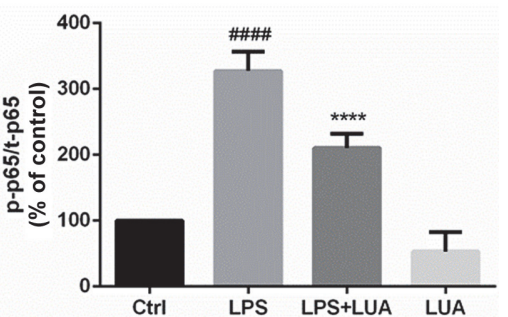

LPS+LUA
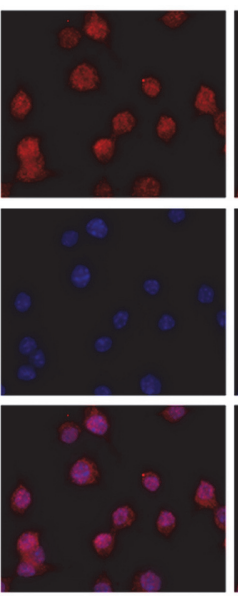

LUA
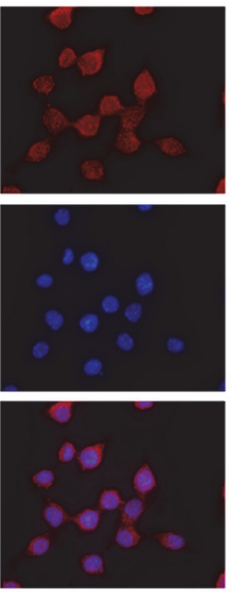

Fig. 8. Effects of LUA on LPS-induced NF-kB signaling pathway in RAW264.7 cells. (A) Cells were incubated with LPS for $30 \mathrm{~min}$ in the absence or presence of LUA $(15 \mu \mathrm{M})$. Cells lysates were subjected to Western blot for analysis of p-p65 $(n=4)$. The relative abundance of p-p65 to t-p65 was quantified. (B) Cells were incubated with LPS for $1 \mathrm{~h}$ in the absence or presence of LUA $(15 \mu \mathrm{M})$. Nuclear translocation of p 65 was visualized by immunofluorescence analysis with a confocal laser scanning microscope. Data were presented as percentage of control group. The results were displayed as means \pm SD. $\# \# \# p<0.0001$ vs. Ctrl group; ${ }^{* * * *} p<0.0001$ vs. LPS group. Ctrl: control; LUA: LUAAPH-1.

\section{Discussion}

Flavones, as a subclass of flavonoids, have a basic structure comprising two aromatic rings (A and B) connected through a heterocyclic C-ring. They differ from other flavonoids via the formation of a double bond between C2 and $\mathrm{C} 3$, following a carbonyl group at the $\mathrm{C} 4$ position on the $\mathrm{C}$-ring. In addition, the absence of a hydroxyl group at the $\mathrm{C} 3$ position of $\mathrm{C}$-ring distinguishes flavones from flavonols, another subgroup of flavonoids [42]. Luteolin is a flavone compound characterized by a hydroxyl group attached at the $\mathrm{C} 5, \mathrm{C} 7, \mathrm{C} 3{ }^{\prime}$, and $\mathrm{C} 4{ }^{\prime}$ positions of the flavone skeleton. Therefore, B-ring of luteolin, with a catechol structure, can be methylated by COMT. Since COMT can only catalyze the substitution of one hydroxyl in catecholic substrates by one O-methyl group from Sadenosyl-L-methionine, two methylated metabolites of luteolin can be formed, which are called chrysoeriol (3'O-methylated luteolin) and diosmetin (4'-O-methylated luteolin). However, some studies have revealed that COMT preferred the methylation for 4'-hydroxyl position to 3'-hydroxyl position of luteolin in human primary hepatocyte, HepG2 cells, human liver S9, and rat tissues [28, 29, 31]. The mechanism for regioselectivity of luteolin might be attributed to the more stable binding mode for 4'-O-methylation of luteolin than that for 3'-Omethylation, which was based on combined theoretical investigations including molecular dynamics simulations, ab initio calculations, and quantum mechanics/molecular mechanics computations [43]. It has been reported that COMT-mediated methylation is responsible for the inactivation of endogenous catecholamine neurotransmitters $[23,44,45]$. Current studies have also observed decreased anti-inflammation ability of methylated luteolin metabolites [31], implying the necessity to improve the chemical structure of luteolin for higher bioactivity considering the methylated metabolism in vivo.

AAPH is a water-soluble azo compound that thermally and spontaneously decomposes to yield nitrogen gas (N2) and two carbon-centered radicals (R.). The carbon radicals could either recombine to generate stable products (RR) or react rapidly and directly with molecule oxygen to yield peroxyl radicals (ROO•) [46], which are implicated in the damage of lipids, proteins, DNA and other cellular constituents [47]. Luteolin is also involved in free radical scavenging and has been reported to be capable of eliminating free radicals generated by AAPH [48, 49], which is a peroxy radical generator for antioxidant capacity assays such as the oxygen radical absorbance capacity assay (ORAC) [50]. We measured the ORAC values of luteolin and found that it had potent activity with 


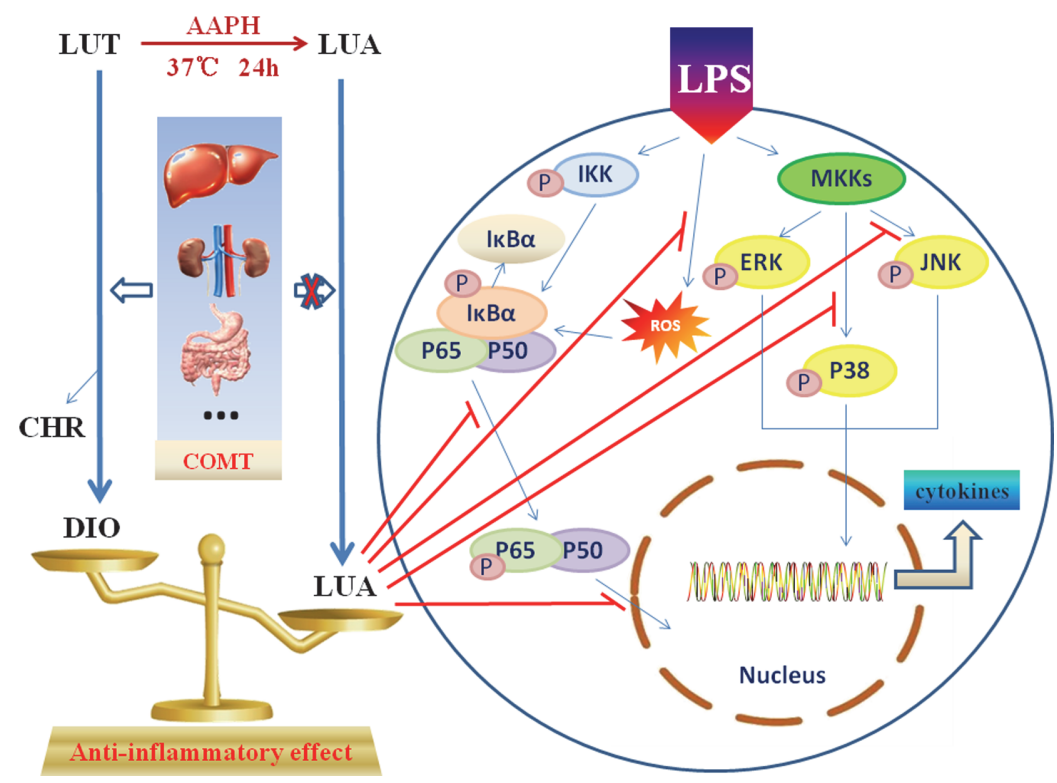

Fig. 9. A schematic model that proposes the synthesis procedure, advantages as well as the potential contribution of LUA in anti-inflammatory signaling pathways in LPS-activated RAW264.7 macrophages. LUT: luteolin; LUA: LUAAPH-1; AAPH: 2,2'-azobis(2-amidinopropane) dihydrochloride; DIO: diosmetin; CHR: chrysoeriol;

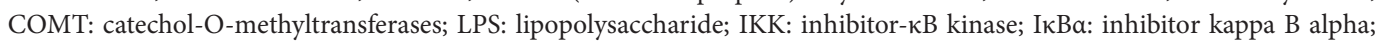
MKKs: MAPK kinases; ROS: reactive oxygen species.

ORAC value of 6.8 [51]. We further characterized the reaction products of luteolin and AAPH and isolated LUA as the major product. This new compound attracted our attention to a great deal because without affecting the skeleton of flavone, the hydroxyl group at the 3' position was substituted by an ester. Therefore, this flavone will not be methylated by COMT at 3 ' and is likely to exhibit its function in the original form in the body.

Flavones have a long tradition of being employed as an anti-inflammatory remedy $[9,52,53]$. It has been suggested that 4'-hydroxyl substituent on the B-ring is tightly related to the anti-inflammatory effect of flavonoids [54]. The LPS-activated RAW264.7 macrophage inflammatory model is widely used in the research of inflammation because macrophage is the main cell that regulates the inflammatory response with LPS as an important trigger. A variety of cytokines participate in the inflammatory response after the stimulation of LPS in RAW264.7 cells. We further investigated the effects of LUA on inflammation in RAW264.7 cells. As expected, with no cytotoxicity up to the concentration of $20 \mu \mathrm{M}$, LUA dose-dependently inhibited the production of NO in macrophages stimulated with LPS. NO production was also compared after LPS treatment in the presence of luteolin, diosmetin and LUA at the concentration of $15 \mu \mathrm{M}$. It was found that although treatment with luteolin inhibited LPS-induced NO production to a large extent, its methylated derivative diosmetin was not as effective as luteolin, indicating the much weaker anti-inflammatory effect of luteolin in the metabolic environment of the organism. There results were in agreement with previous research [31]. However, compared with diosmetin, LUA was superior in the suppression of NO, suggesting the possible better anti-inflammatory effect of LUA than luteolin in vivo. The reduction of NO production was likely attributed to the transcriptional level as indicated by the attenuated expression of iNOS mRNA and protein expression. In addition, LUA also substantially down regulated the production of pro-inflammatory molecules including COX-2, IL- $1 \beta$, IL-6, IL-8, and IFN- $\beta$, which was similar to the anti-inflammatory effects of luteolin [55-58].

Notably, accumulated evidence indicates that inflammation and oxidative stress are highly related and orchestrated between each other, creating a vicious cycle in various pathophysiological conditions [59-61]. On one hand, inflammatory cells can liberate more ROS at the inflammatory lesion, leading to exaggerated oxidative stress $[59,62]$. On the other hand, a number of ROS can provoke an intracellular signaling cascade that augments pro-inflammatory gene expression. Earlier studies have demonstrated that luteolin suppresses the ROS generation [15], thus regulating the homeostasis between oxidants and antioxidants [18, 20,63-65]. In our study, LPS-induced inflammation was accompanied by large amounts of ROS generation in the cells as reported [66, 67], whereas ROS were prominently repressed by LUA, suggesting that LUA also exerted its anti-inflammatory effect by removing intracellular ROS.

It is well known that MAPK signaling pathways play crucial roles in regulating the inflammatory signaling from cell surface to nucleus and coordinating induction of different genes encoding for inflammatory mediators [41, 68-70]. It remains controversial whether luteolin is responsible for the inhibition of MAPK signaling pathway in RAW cells stimulated by LPS. Chen et al. discovered that treatment of RAW cells with luteolin only decreased JNK phosphorylation and its downstream signaling [71]. Intriguingly, instead of inhibiting MAPK pathway, Lee et al. found that luteolin upregulated the phosphorylation of these enzymes in LPS-induced RAW cells [55]. Our results 
showed that exposure of RAW cells to LPS increased the phosphorylation of p38, ERK and JNK respectively. We further demonstrated that LUA markedly suppressed the phosphorylation of JNK and p38 stimulated by LPS without changing the total protein levels of MAPKs, indicating that LUA specifically inhibited the phosphorylation of JNK and p38 but not the synthesis of these two MAPKs. In contrast, LUA did not affect the phosphorylation of ERK elicited by LPS. Thus, we presumed that LUA-mediated suppression of LPS-induced inflammation in RAW cells was JNK and p38 dependent.

As an important nuclear transcription factor, NF- $\mathrm{BB}$ is also a key mediator of inflammation. In the inactive mode, the NF- $\kappa \mathrm{B}$ dimer (p50/p65) exists in the cytosol and is bound to I $\mathrm{B} a$, an inhibitory protein. The presence of several stimuli, including LPS, leads to the phosphorylation and degradation of IкBa, followed by p65 phosphorylation and translocation of NF- $\kappa$ B into the nucleus, where NF- $\kappa$ B subsequently binds to corresponding sites and activates the transcription of many pro-inflammatory genes ultimately [72-75]. It has been noted that LPS-induced ROS generation can also transduce signals to activate NF- $\kappa B$ signaling cascades in LPS-stimulated macrophages [76]. Several previous studies have provided evidence that luteolin mediated the anti-inflammatory effect by blocking the NF- $\mathrm{KB}$ signaling pathway $[55,57,77]$. In line with these findings, in the present study, LUA significantly abolished LPS-induced p65 phosphorylation and the subsequent accumulation of $\mathrm{p} 65$ in the nucleus without affecting the expression of total p65. Therefore, it is expected that the anti-inflammatory property of LUA also attributed to the inhibition of NF- $\kappa B$ signaling pathway.

In conclusion, we described a novel compound (LUAAPH-1) generated by the chemical reaction between luteolin and AAPH, which was characterized by several unique features. First of all, compared to luteolin, LUA could escape COMT-catalyzed methylation, thus affording the potential to exert its functions in the original form when administrated in the organism. Secondly, the new compound displayed highly superior anti-inflammatory activities than the methylated derivative of luteolin in macrophages and the underlying mechanisms might be the inhibition of LPS-activated JNK, P38 and NF- $\mathrm{kB}$ signaling pathways (Fig. 9). The findings suggested the new compound to be a potentially promising therapy in inflammation disorders and related diseases. However, more investigation into the therapeutic effects of the new compound in vivo are still required for the potential clinical applications.

\section{Acknowledgments}

This work was supported by the Science and Technology Project of Changzhou City (Grant No. CE20215028).

\section{Conflicts of Interest}

The authors have no financial conflicts of interest to declare.

\section{References}

1. Yeom M, Kim JH, Min JH, Hwang MK, Jung HS, Sohn Y. 2015. Xanthii fructus inhibits inflammatory responses in LPS-stimulated RAW 264.7 macrophages through suppressing NF-kappaB and JNK/p38 MAPK. J. Ethnopharmacol. 176: 394-401.

2. Erwig LP, Rees AJ. 1999. Macrophage activation and programming and its role for macrophage function in glomerular inflammation. Kidney Blood Press. Res. 22: 21-25

3. Hoffmann JA, Kafatos FC, Janeway CA, Ezekowitz RA. 1999. Phylogenetic perspectives in innate immunity. Science 284: $1313-1318$.

4. King GL. 2008. The role of inflammatory cytokines in diabetes and its complications. J. Periodontol. 79: 1527-1534.

5. Kundu JK, Surh YJ. 2008. Inflammation: gearing the journey to cancer. Mutat. Res. 659: 15-30.

6. Manzi S, Wasko MC. 2000. Inflammation-mediated rheumatic diseases and atherosclerosis. Ann. Rheum. Dis. 59: 321-325.

7. Balkwill F, Mantovani A. 2001. Inflammation and cancer: Back to Virchow? Lancet 357: 539-545.

8. Manach C, Scalbert A, Morand C, Remesy C, Jimenez L. 2004. Polyphenols: food sources and bioavailability. Am. J. Clin. Nutr. 79: 727-747.

9. Feng X, Qin H, Shi Q, Zhang Y, Zhou F, Wu H, et al. 2014. Chrysin attenuates inflammation by regulating M1/M2 status via activating PPARgamma. Biochem. Pharmacol. 89: 503-514.

10. Ruiz PA, Haller D. 2006. Functional diversity of flavonoids in the inhibition of the proinflammatory NF-kappaB, IRF, and Akt signaling pathways in murine intestinal epithelial cells. J. Nutr. 136: 664-671.

11. Qian LB, Wang HP, Chen Y, Chen FX, Ma YY, Bruce IC, et al. 2010. Luteolin reduces high glucose-mediated impairment of endothelium-dependent relaxation in rat aorta by reducing oxidative stress. Pharmacol. Res. 61: 281-287.

12. Jennings A, Welch AA, Spector T, Macgregor A, Cassidy A. 2014. Intakes of anthocyanins and flavones are associated with biomarkers of insulin resistance and inflammation in women. J. Nutr. 144: 202-208.

13. Lin Y, Shi R, Wang X, Shen HM. 2008. Luteolin, a flavonoid with potential for cancer prevention and therapy. Curr. Cancer Drug Targets 8: 634-646.

14. Liu CW, Lin HW, Yang DJ, Chen SY, Tseng JK, Chang TJ, et al. 2016. Luteolin inhibits viral-induced inflammatory response in RAW264.7 cells via suppression of STAT1/3 dependent NF-kappaB and activation of HO-1. Free Radic. Biol. Med. 95: 180-189.

15. Wolfle U, Esser PR, Simon-Haarhaus B, Martin SF, Lademann J, Schempp CM. 2011. UVB-induced DNA damage, generation of reactive oxygen species, and inflammation are effectively attenuated by the flavonoid luteolin in vitro and in vivo. Free Radic. Biol. Med. 50: 1081-1093.

16. Pratheeshkumar P, Son YO, Divya SP, Roy RV, Hitron JA, Wang L, et al. 2014. Luteolin inhibits Cr(VI)-induced malignant cell transformation of human lung epithelial cells by targeting ROS mediated multiple cell signaling pathways. Toxicol. Appl. Pharmacol. 281: 230-241.

17. Kwon EY, Jung UJ, Park T, Yun JW, Choi MS. 2015. Luteolin attenuates hepatic steatosis and insulin resistance through the interplay between the liver and adipose tissue in mice with diet-induced obesity. Diabetes 64: 1658-1669.

18. Seelinger G, Merfort I, Schempp CM. 2008. Anti-oxidant, anti-inflammatory and anti-allergic activities of luteolin. Planta Med. 74: $1667-1677$

19. Hu C, Kitts DD. 2004. Luteolin and luteolin-7-O-glucoside from dandelion flower suppress iNOS and COX-2 in RAW264.7 cells. Mol. Cell. Biochem. 265: 107-113. 
20. Wu W, Li D, Zong Y, Zhu H, Pan D, Xu T, et al. 2013. Luteolin inhibits inflammatory responses via p38/MK2/TTP-mediated mRNA stability. Molecules 18: 8083-8094.

21. Fei J, Liang B, Jiang C, Ni H, Wang L. 2019. Luteolin inhibits IL-1beta-induced in $\mathrm{fl}$ ammation in rat chondrocytes and attenuates osteoarthritis progression in a rat model. Biomed. Pharmacother. 109: 1586-1592.

22. Chen Z, Tu M, Sun S, Kong S, Wang Y, Ye J, et al. 2012. The exposure of luteolin is much lower than that of apigenin in oral administration of Flos Chrysanthemi extract to rats. Drug Metab. Pharmacokinet. 27: 162-168.

23. Guldberg HC, Marsden CA. 1975. Catechol-O-methyl transferase: pharmacological aspects and physiological role. Pharmacol. Rev. 27: $135-206$

24. Nissinen E, Tuominen R, Perhoniemi V, Kaakkola S. 1988. Catechol-O-methyltransferase activity in human and rat small intestine. Life Sci. 42: 2609-2614.

25. Schultz E. 1991. Catechol-O-methyltransferase and aromatic L-amino acid decarboxylase activities in human gastrointestinal tissues. Life Sci. 49: 721-725.

26. Mannisto PT, Kaakkola S. 1999. Catechol-O-methyltransferase (COMT): biochemistry, molecular biology, pharmacology, and clinical efficacy of the new selective COMT inhibitors. Pharmacol. Rev. 51: 593-628.

27. Meloto CB, Segall SK, Smith S, Parisien M, Shabalina SA, Rizzatti-Barbosa CM, et al. 2015. COMT gene locus: new functional variants. Pain 156: 2072-2083.

28. Chen ZJ, Dai YQ, Kong SS, Song FF, Li LP, Ye JF, et al. 2013. Luteolin is a rare substrate of human catechol-O-methyltransferase favoring a para-methylation. Mol. Nutr. Food Res. 57: 877-885.

29. Chen Z, Chen M, Pan H, Sun S, Li L, Zeng S, et al. 2011. Role of catechol-O-methyltransferase in the disposition of luteolin in rats. Drug Metab. Dispos. 39: 667-674.

30. Wang L, Chen Q, Zhu L, Li Q, Zeng X, Lu L, et al. 2017. Metabolic Disposition of Luteolin is mediated by the interplay of UDPGlucuronosyltransferases and Catechol-O-Methyltransferases in rats. Drug Metab. Dispos. 45: 306-315.

31. Ha SK, Lee JA, Cho EJ, Choi I. 2017. Effects of Catechol O-Methyl Transferase inhibition on anti-inflammatory activity of luteolin metabolites. J. Food Sci. 82: 545-552.

32. Parashos SA, Wielinski CL, Kern JA. 2004. Frequency, reasons, and risk factors of entacapone discontinuation in Parkinson disease. Clin. Neuropharmacol. 27: 119-123.

33. Assal F, Spahr L, Hadengue A, Rubbia-Brandt L, Burkhard PR. 1998. Tolcapone and fulminant hepatitis. Lancet 352: 958

34. Olanow CW. 2000. Tolcapone and hepatotoxic effects. Tasmar advisory panel. Arch. Neurol. 57: 263-267.

35. DeLeo FR, Renee J, McCormick S, Nakamura M, Apicella M, Weiss JP, et al. 1998. Neutrophils exposed to bacterial lipopolysaccharide upregulate NADPH oxidase assembly. J. Clin. Invest. 101: 455-463.

36. Keller JN, Hanni KB, Gabbita SP, Friebe V, Mattson MP, Kindy MS. 1999. Oxidized lipoproteins increase reactive oxygen species formation in microglia and astrocyte cell lines. Brain Res. 830: 10-15.

37. Kaminska B. 2005. MAPK signalling pathways as molecular targets for anti-inflammatory therapy--from molecular mechanisms to therapeutic benefits. Biochim. Biophys. Acta. 1754: 253-262.

38. Kim YS, Ahn CB, Je JY. 2016. Anti-inflammatory action of high molecular weight Mytilus edulis hydrolysates fraction in LPSinduced RAW264.7 macrophage via NF-kappaB and MAPK pathways. Food Chem. 202: 9-14.

39. Liu F, Zhang X, Ling P, Liao J, Zhao M, Mei L, et al. 2017. Immunomodulatory effects of xanthan gum in LPS-stimulated RAW 264.7 macrophages. Carbohydr. Polym. 169: 65-74.

40. Shan J, Fu J, Zhao Z, Kong X, Huang H, Luo L, et al. 2009. Chlorogenic acid inhibits lipopolysaccharide-induced cyclooxygenase-2 expression in RAW264.7 cells through suppressing NF-kappaB and JNK/AP-1 activation. Int. Immunopharmacol. 9: $1042-1048$.

41. Ajizian SJ, English BK, Meals EA. 1999. Specific inhibitors of p38 and extracellular signal-regulated kinase mitogen-activated protein kinase pathways block inducible nitric oxide synthase and tumor necrosis factor accumulation in murine macrophages stimulated with lipopolysaccharide and interferon-gamma. J. Infect. Dis. 179: 939-944.

42. Aziz N, Kim MY, Cho JY. 2018. Anti-inflammatory effects of luteolin: a review of in vitro, in vivo, and in silico studies. J. Ethnopharmacol. 225: 342-358.

43. Cao Y, Chen ZJ, Jiang HD, Chen JZ. 2014. Computational studies of the regioselectivities of COMT-catalyzed meta-/para-O methylations of luteolin and quercetin. J. Phys. Chem. B. 118: 470-481.

44. Eisenhofer G, Kopin IJ, Goldstein DS. 2004. Catecholamine metabolism: a contemporary view with implications for physiology and medicine. Pharmacol. Rev. 56: 331-349.

45. Kopin IJ. 1985. Catecholamine metabolism: basic aspects and clinical significance. Pharmacol. Rev. 37: 333-364.

46. Dooley MM, Sano N, Kawashima H, Nakamura T. 1990. Effects of 2,2'-azobis (2-amidinopropane) hydrochloride in vivo and protection by vitamin E. Free Radic. Biol. Med. 9: 199-204.

47. Muraoka S, Miura T. 2002. Protection by estrogens of biological damage by 2,2'-azobis(2-amidinopropane) dihydrochloride. J. Steroid Biochem. Mol. Biol. 82: 343-348.

48. Kanazawa K, Uehara M, Yanagitani H, Hashimoto T. 2006. Bioavailable flavonoids to suppress the formation of 8-OHdG in HepG2 cells. Arch. Biochem. Biophys. 455: 197-203.

49. Edenharder R, Grunhage D. 2003. Free radical scavenging abilities of flavonoids as mechanism of protection against mutagenicity induced by tert-butyl hydroperoxide or cumene hydroperoxide in Salmonella typhimurium TA102. Mutat. Res. 540: 1-18.

50. Huang D, Ou B, Hampsch-Woodill M, Flanagan JA, Prior RL. 2002. High-throughput assay of oxygen radical absorbance capacity (ORAC) using a multichannel liquid handling system coupled with a microplate fluorescence reader in 96-well format. J. Agric. Food Chem. 50: 4437-4444.

51. Zuvela P, David J, Yang X, Huang D, Wong MW. 2019. Non-Linear quantitative structure(-) activity relationships modelling, mechanistic study and in-silico design of flavonoids as potent antioxidants. Int. J. Mol. Sci. 20: 2328.

52. Kumar KJ, Yang HL, Tsai YC, Hung PC, Chang SH, Lo HW, et al. 2013. Lucidone protects human skin keratinocytes against free radical-induced oxidative damage and inflammation through the up-regulation of $\mathrm{HO}-1 / \mathrm{Nrf} 2$ antioxidant genes and downregulation of NF-kappaB signaling pathway. Food Chem. Toxicol. 59: 55-66.

53. Zeinali M, Rezaee SA, Hosseinzadeh H. 2017. An overview on immunoregulatory and anti-inflammatory properties of chrysin and flavonoids substances. Biomed. Pharmacother. 92: 998-1009.

54. Manthey JA, Grohmann K, Guthrie N. 2001. Biological properties of citrus flavonoids pertaining to cancer and inflammation. Curr. Med. Chem. 8: 135-153.

55. Lee JO, Jeong D, Kim MY, Cho JY. 2015. ATP-binding pocket-targeted suppression of Src and Syk by luteolin contributes to its antiinflammatory action. Mediators Inflamm. 2015: 967053.

56. Park CM, Jin KS, Lee YW, Song YS. 2011. Luteolin and chicoric acid synergistically inhibited inflammatory responses via inactivation of PI3K-Akt pathway and impairment of NF-kappaB translocation in LPS stimulated RAW 264.7 cells. Eur. J. Pharmacol. 660: 454-459.

57. Park CM, Song YS. 2013. Luteolin and luteolin-7-O-glucoside inhibit lipopolysaccharide-induced inflammatory responses through modulation of NF-kappaB/AP-1/PI3K-Akt signaling cascades in RAW 264.7 cells. Nutr. Res. Pract. 7: 423-429. 
58. Park CM, Park JY, Noh KH, Shin JH, Song YS. 2011. Taraxacum officinale Weber extracts inhibit LPS-induced oxidative stress and nitric oxide production via the NF-kappaB modulation in RAW 264.7 cells. J. Ethnopharmacol. 133: 834-842.

59. Biswas SK. 2016. Does the Interdependence between oxidative stress and inflammation explain the antioxidant paradox? Oxid. Med. Cell. Longev. 2016: 5698931.

60. Gao B, Tsukamoto H. 2016. Inflammation in alcoholic and nonalcoholic fatty liver disease: friend or foe? Gastroenterology 150: 1704-1709.

61. Lam P, Cheung F, Tan HY, Wang N, Yuen MF, Feng Y. 2016. Hepatoprotective effects of chinese medicinal herbs: a focus on antiinflammatory and anti-oxidative activities. Int. J. Mol. Sci. 17: 465.

62. Ambade A, Mandrekar P. 2012. Oxidative stress and inflammation: essential partners in alcoholic liver disease. Int. J. Hepatol. 2012: 853175 .

63. Robak J, Shridi F, Wolbis M, Krolikowska M. 1988. Screening of the influence of flavonoids on lipoxygenase and cyclooxygenase activity, as well as on nonenzymic lipid oxidation. Pol. J. Pharmacol. Pharm. 40: 451-458.

64. Brown JE, Rice-Evans CA. 1998. Luteolin-rich artichoke extract protects low density lipoprotein from oxidation in vitro. Free Rradic. Res. 29: 247-255

65. Zhang T, Wu W, Li D, Xu T, Zhu H, Pan D, et al. 2014. Anti-oxidant and anti-apoptotic effects of luteolin on mice peritoneal macrophages stimulated by angiotensin II. Int. Immunopharmacol. 20: 346-351.

66. Xu Q, Liu M, Liu Q, Wang W, Du Y, Yin H. 2017. The inhibition of LPS-induced inflammation in RAW264.7 macrophages via the PI3K/Akt pathway by highly $N$-acetylated chitooligosaccharide. Carbohydr. Polym. 174: 1138-1143.

67. Lee JA, Song HY, Ju SM, Lee SJ, Kwon HJ, Eum WS, et al. 2009. Differential regulation of inducible nitric oxide synthase and cyclooxygenase-2 expression by superoxide dismutase in lipopolysaccharide stimulated RAW 264.7 cells. Exp. Mol. Med. 41: 629637.

68. Bhat NR, Zhang P, Lee JC, Hogan EL. 1998. Extracellular signal-regulated kinase and p38 subgroups of mitogen-activated protein kinases regulate inducible nitric oxide synthase and tumor necrosis factor-alpha gene expression in endotoxin-stimulated primary glial cultures. J. Neurosci. 18: 1633-1641.

69. Hambleton J, Weinstein SL, Lem L, DeFranco AL. 1996. Activation of c-Jun N-terminal kinase in bacterial lipopolysaccharidestimulated macrophages. Proc. Nat. Acad. Sci. USA 93: 2774-2778.

70. Hommes DW, Peppelenbosch MP, van Deventer SJ. 2003. Mitogen activated protein (MAP) kinase signal transduction pathways and novel anti-inflammatory targets. Gut 52: 144-151.

71. Chen D, Bi A, Dong X, Jiang Y, Rui B, Liu J, et al. 2014. Luteolin exhibits anti-inflammatory effects by blocking the activity of heat shock protein 90 in macrophages. Biochem. Biophys. Res. Commun. 443: 326-332.

72. Milanovic M, Kracht M, Schmitz ML. 2014. The cytokine-induced conformational switch of nuclear factor kappaB p65 is mediated by 65 phosphorylation. Biochem. J. 457: 401-413.

73. Qian Z, Wu Z, Huang L, Qiu H, Wang L, Li L, et al. 2015. Mulberry fruit prevents LPS-induced NF-kappaB/pERK/MAPK signals in macrophages and suppresses acute colitis and colorectal tumorigenesis in mice. Sci. Rep. 5: 17348.

74. Perkins ND. 2007. Integrating cell-signalling pathways with NF-kappaB and IKK function. Nature reviews. Mol. Cell Biol. 8: 49-62.

75. Karin M, Ben-Neriah Y. 2000. Phosphorylation meets ubiquitination: the control of NF-[kappa]B activity. Ann. Rev. Immunol. 18: 621-663.

76. Gloire G, Legrand-Poels S, Piette J. 2006. NF-kappaB activation by reactive oxygen species: fifteen years later. Biochem. Pharmacol. 72: $1493-1505$.

77. Sung J, Lee J. 2015. Anti-inflammatory activity of Butein and Luteolin through suppression of NFkappaB activation and induction of heme oxygenase-1. J. Med. Food 18: 557-564.

78. Xia MZ, Liang YL, Wang H, Chen X, Huang YY, Zhang ZH, et al. 2012. Melatonin modulates TLR4-mediated inflammatory genes through MyD88- and TRIF-dependent signaling pathways in lipopolysaccharide-stimulated RAW264.7 cells. J. Pineal Res. 53: 325334. 